\title{
Sentido de vida y salud mental
}

\author{
Sense of life and mental health
}

\author{
David Alberto Zamudio Flores ${ }^{1}$ \\ Universidad Continental
}

Recibido: $27-05-21$

Aceptado: $12-11-21$

Publicado: $23-12-21$

\section{Resumen}

El presente ensayo aborda la importancia y trascendencia de la concepción de sentido de vida y su posterior enriquecimiento a lo largo de la vida de una persona, así como su implicancia en un proceso terapéutico, todo ello como parte esencial del fortalecimiento de la salud mental. Reflexión y análisis del autor a partir de la revisión de fuentes bibliográficas de manera metódica y sistemática, artículos, reportes de investigación y revistas científicas de psicología indexadas en bases de datos electrónicas, así como, la utilización de libros físicos y digitales. En conclusión, poseer un sentido de vida sano, inspirador e intrínseco permite fortalecer las bases de la salud mental, así también, contribuye significativamente en todo proceso terapéutico, ejerciendo prevención e intervención oportuna.

Palabras clave: Sentido de vida; salud mental; proceso terapéutico.

\begin{abstract}
This essay addresses the importance and significance of the conception of the meaning of life and its subsequent enrichment throughout a person's life, as well as its implication in a therapeutic process, all as an essential part of strengthening health mental. Reflection and analysis of the author from the review of bibliographic sources in a methodical and systematic way, articles, research reports and scientific psychology journals indexed in electronic databases, as well as, the use of physical and digital books. Having a healthy, inspiring and intrinsic sense of life allows strengthening the foundations of mental health, as well as contributing significantly to the entire therapeutic process, exercising prevention and timely intervention.
\end{abstract}

Keywords: Sense of life; mental health; therapeutic process.

1 Docente de las Facultades de Humanidades y Ciencias de la Salud de la Universidad Continental, Perú.

Autor para correspondencia: dzamudio@continental.edu.pe ORCID: https://orcid.org/0000-0002-4977-1039

(C) Los autores. Este artículo es publicado por la Revista de Investigación en Psicología de la Facultad de Psicología, Universidad Nacional Mayor de San Marcos. Este es un artículo de acceso abierto, distribuido bajo los términos de la licencia Creative Commons Atribución 4.0 Internacional (CC BY 4.0) [https://creativecommons.org/licenses/by/4.0/deed.es] que permite el uso, distribución y reproducción en cualquier medio, siempre que la obra original sea debidamente citada de su fuente original. 
El sentido de vida es la concepción que tiene la persona que le permite encontrar el significado a su existencia, la ausencia o incorrecta interpretación de este concepto puede originar diversas perturbaciones a nivel psicológico, por el contrario, comprender su importancia y enriquecerlo a lo largo de la vida posibilita mayor solvencia para enfrentar crisis a nivel mental, emocional y sentimental, crisis que si no son debidamente tratadas o abordadas derivan en trastornos de personalidad, ansiedad y de estado de ánimo, es decir, la importancia de encontrar nuestro sentido de vida, más aún, en un tratamiento psicoterapéutico, contribuye a superar diversas afecciones que son lesivas para la salud mental. Martínez (2012) refiere que el sentido de vida es la percepción cognitiva y afectiva de valores que posibilitan a un individuo a comportarse de una manera determinada, ante un evento o la vida, brindando al individuo coherencia e identidad personal. Por lo tanto, Lukas (2005) refiere que el Sentido de Vida es la búsqueda de una persona por darle valor a su existencia, así también, menciona que es una búsqueda individual, no puede influir nadie más, ya que cuando este sentido de vida llega a ser enriquecido, se logra la realización a nivel personal.

De tal manera que, Luna (2011) especifica que el individuo ejerce su vida en tensión, en búsqueda de valores y es así que encontrará significados existenciales, lo que forjará su sentido de vida.

No tener un sentido de vida equivaldría a un vacío existencial el cual se representa fundamentalmente por una condición de aburrimiento y hartazgo (Frankl, 2004). Así mismo, el filósofo Buber (1969), mencionó que el ser humano vive una especie de capitalismo que genera que los seres humanos se equivoquen o confundan creyendo que la carencia de algún objeto material representa ese vacío existencial, y por ello esa necesidad compulsiva de comprar o tener para sentirse felices, a partir de todo ello se produce en el individuo la imposibilidad de comprenderse a sí mismo en sus momentos de vacío y soledad, momentos claves para que pueda construir, desarrollar y enriquecer su autoconocimiento personal. Bauman (2008) sostiene que ser individuos conscientes en una sociedad en la que la publicidad y las ofertas marcan las pautas de trascendencia generan confusión, marcas como etiquetas son las que definen el reconocimiento, aspiraciones, metas, todo ello como reglas que posibilitan ser reconocido, y que marcan la identidad de un individuo o grupo.

Nos podemos percatar que actualmente nuestra sociedad moderna en su mayoría no destaca la importancia de las relaciones y vínculos humanos, por el contrario, las despersonaliza, buscando la inmediatez y la conveniencia, es decir, dejando de lado la calidez de comunidades antiguas, menos ostentosas y más avocadas a priorizar los sentimientos y afectos (Savater, 2007). Así también, Gottfried (2016) manifiesta que, como parte de uno de los problemas a nivel individual y social característicos de la última mitad del siglo XX es el vacío existencial, producto de no tener un sentido de vida, propio de sociedades 
post modernas, lo cual, evidentemente no era característico en comunidades del pasado.

El logoterapeuta García (2010), tipifica curiosamente a nuestra sociedad actual, denominándola "mundo Popeye", esto debido a que la fuerza interna de muchas personas proviene de la lata de cerveza, de pastillas, de medicamentos que nos dan la ilusoria creencia de ser felices, de automóviles magníficos que supuestamente determinan nuestra valía como seres humanos o delimitan valor social, en general de cosas, entonces, estas "espinacas" que revitalizan o empoderan al ser humano, haciendo alusión a su calificación de la sociedad, es lo que genera una falsa y efímera creencia de creernos valiosos en función a lo que adquirimos, compramos, mostramos o consumimos, pero, internamente el vacío existencial se está haciendo cada vez más grande debido a la sobredimensionada importancia de lo externo, de lo material.

Como parte del vertiginoso y acelerado avance en ciencia y tecnología conseguido por los seres humanos evidenciado principalmente en las metrópolis, el hecho de preocuparse por sobrevivir, salvaguardarse o conseguir alimento en un gran número de personas ya no es preocupación latente a diferencia de otras épocas, ya que a pesar de sus limitaciones e imperfecciones en la gran mayoría de sociedades se trabaja por solventar esas preocupaciones, por lo que, la preocupación de la humanidad es hallar la respuesta al sentido de la vida, la cual es una necesidad primordialmente humana (Velasco, 2004). Y en base al tema de necesidades, es pertinente destacar lo que refiere Malos (2005) en su teoría, acerca que el modelo de la jerarquía de necesidades humanas, la cual abarca y establece un conjunto de necesidades humanas jerarquizadas, el concepto más destacable es la autoactualización, o la necesidad humana más elevada, la que se representa a través de la satisfacción justificada en un sentido de vida mediante el desarrollo enriquecedor de una actividad.

En cuanto al desarrollo del sentido de vida, el ser humano no solo busca desarrollarlo a través de sus pensamientos y acciones individualmente, puesto que también busca que las demás también lo lleguen a desarrollar, es decir, constituye vida para la misma persona como para la familia y la sociedad, derivando en enriquecimiento personal y grupal, lo cual contribuye no solo a nivel de la salud mental individual sino también colectiva o social (Salomón, 2015). Así también, Luna (2011) refiere que Frankl desarrolla la categoría de los valores representados en: Valores de Creación, que es buscar el sentido por medio de lograr los proyectos personales; Valores de Experiencia, cuando el individuo logra el sentido por medio de sus vivencias o de experimentar el valor de otro individuo, a partir de la afectividad; Valores de Actitud, que implica descubrir el sentido sólo cuando el ser humano es capaz de escoger y ejercer la actitud con la que afronta situaciones a nivel social. 


\section{DESARROLLO}

\section{Sucinto panorama actual}

El vivir en una sociedad tan hedonista, donde se sobrevalora la inmediatez y la felicidad se traduce en abundancia, ha distanciado al ser humano de su sentido de existencia, confundiéndolo, y tratando de encontrar sentido en el placer efímero, en lo material, en lo físico, aunado a todo ello, la pérdida de sus creencias religiosas, vitales para dar sentido a la vida y a la muerte, genera vacíos existenciales, estilos de vida sin valores, lo cual degenera en una asfixiante soledad, infelicidad, y en la aparición de trastornos mentales que debilitan la calidad de vida del individuo y por ende de la sociedad.

Uno de los factores, que aún hoy no se acepta su potente efecto y que ha dificultado el desarrollo del sentido de vida es la publicidad incorrectamente ejercida y transmitida por diversos medios, publicidad mal enfocada y agresiva que vivimos hoy en día y que desde muy temprana edad bombardea al individuo haciendo que elabore creencias sin fundamento acerca del bienestar personal y psicológico, se puede observar aproximadamente desde la década de los sesenta del siglo pasado como existen masas de consumidores cautivados, obsesionados y dependientes en su vida diaria por lo que establece la publicidad, la moda, la hiper tecnología, siendo totalmente cegados e indiferentes a la importancia de la interiorización y reflexión como parte del sentido de vida, tal como plantea el filósofo y sociólogo polaco Bauman (2007).

Así también, Gómez (2013) sostiene que el advenimiento de mayores alternativas de distracción por intermedio de internet genera narcisismo, así como acciones que no profundizan, no ahondan en el conocimiento, los individuos se sienten muy cómodos con los resúmenes y la inmediatez, buscando siempre la definición o explicación más escueta, existe pereza para pensar y reflexionar, lo cual genera una tergiversación en cuanto a la búsqueda del sentido de vida, ya que todo debe ser fácil, inmediato y no requerir esfuerzo, es decir, se enarbola la escasez y la simplicidad, lo cual afecta la concepción de un proyecto de vida y el esfuerzo y constancia que ello implica.

\section{La búsqueda del sentido de vida}

Es imprescindible entender y priorizar el sentido de vida que permita generar o, en el mejor de los casos, preservar la salud mental, representada por la esperanza, proyectos de vida sanos e inspiradores, bienestar personal y social, y para ello es sustancial que de una vez por todas la sociedad sea un ente activo, pero positivo, en cuanto a la consecución de ese objetivo o meta.

Este ferviente deseo por encontrar nuestro sentido de vida, y si lo hemos hallado, por fortalecerlo en el transcurso de nuestra existencia, permiten ser 
una fuente de motivación constante en nuestra vida y por consecuencia superar crisis, adversidades o dificultades cuando se nos presenten en nuestra vida de una manera resiliente. Frankl (2011) entiende que, esta voluntad y esfuerzo por hallar el significado de nuestra propia existencia compone la principal fortaleza con respecto a la motivación del ser humano, vinculada estrechamente con la espiritualidad y la fe.

Según Viktor Frankl (1999) el anhelo por encontrar este sentido de vida se encuentra presente en todas las personas, ya sea en mayor o en menor medida. Cabe señalar que, el sentido de vida no es idéntico para todas las personas, puesto que dependerá de los objetivos, capacidades, y circunstancias que presenta cada ser humano y que por el cual busca un significado a su existencia. Este significado cada persona lo debe encontrar, es una búsqueda personal que no se puede delegar a otro u otros, pero, con ayuda de un proceso terapéutico se puede motivar a encontrarlo y fortalecerlo, a partir de brindar claves, herramientas y estrategias que contribuyan en su hallazgo y posterior enriquecimiento personal.

\section{Influencia del sentido de vida en la salud mental}

Básicamente, si es que existe el deseo de hallar un significado en la vida o tenerlo, ello implica salud mental, este bienestar psicológico producto de haberlo hallado es muy relevante cuando se analiza el estado de salud mental de cualquier individuo en diferentes periodos o etapas de su vida, ya que, es importante tener en consideración que el sentido va cambiando en el transcurso de la vida, y ante ello no solo está involucrado el desarrollo físico, sino, principalmente el desarrollo psicológico, ya que cambian nuestras aspiraciones, metas y objetivos, en las diferentes etapas de nuestra vida priorizando o incorporando valores.

Se presentan circunstancias que denotan falta de sentido de vida o lo que se denomina también como vacío existencial, entre estas están: el aventurismo, el cual implica involucrarse fanáticamente a alguna causa o labor, sin importar su valor o reflexionar sobre su importancia o trascendencia; el nihilismo, representada por la carencia de sentido de vida y por consiguiente desacreditar las actividades de propósito personal en los demás; forma vegetativa, catalogado como un estado de hartazgo, aburrimiento e indiferencia que conlleva a depresión (Yalom, 1984).

La carencia de sentido de vida está fuertemente vinculada con el tiempo libre u ocio, ya que esta despreocupación o falta de obligaciones nos pone cara a cara con nuestra libertad personal en un mundo actual en el que el ser humano se enfrenta a las circunstancias de su existencia sin poseer un significado cósmico o religioso de la vida, alejado completamente de la naturaleza y su complementariedad con esta. Frankl (1999) refiere que la inexistencia de significado de vida es una clara advertencia de desajuste emocional. En nuestros días claramente se ve la incidencia de personas que sufren de vacío existencial o no encuentran sentido a su vida, 
estas características corresponden a la nooneurosis, acuñado por el mismo Frankl, y que hace referencia a la neurosis originada ante la falta de sentido de vida, y se ve plasmado o representado en diversas afecciones tales como la drogadicción, ludopatías, depresión, oniomanía, distrés, entre otras.

Según Frankl (1994), la felicidad se logra teniendo un motivo por el cual vivir añadido a la importancia de los valores, vale decir, que tanto la teoría de Viktor Frankl como la psicología positiva de Seligman (2003) tienen vínculos y similitudes con respecto a lo que se denomina como las tres fases en las que se encontrará una persona en función a la felicidad y su sentido de vida. La primera fase denominada vacío existencial y vida placentera, representada por el carente sentido de existencia, enfocada en el placer de la inmediatez, sin trascendencia y en ausencia de valores que deriva en la deshumanización, por ello, Baleriano, Pinzón, GaonaPérez y Hernández (2015), proponen enfatizar en los valores los cuales deben ser establecidos y fortificados desde los diversos entornos en los que se desarrolla el ser humano tales como la familia, escuela y sociedad. Una segunda fase denominada buena vida y voluntad de sentido, Frankl (1999) sostiene que es trascendental los valores como voluntad de sentido y que estos deben ser vivenciales, es decir, dejar de priorizar solo la retórica, sino que principalmente se enseñe con el ejemplo y que a partir de cómo se afronta el sufrimiento se realzará los valores de actitud, así también, Seligman (2003), refiere que debe de haber un compromiso en obtener una buena vida en área laboral, sentimental y personal, y para ello se debe hacer uso de las fortalezas y virtudes, lo cual proveerá de dominio personal y una clara visión optimista ante diversas circunstancias de la vida. Tercera fase, catalogada como vida con significado y sentido último, este último, según Seligman (2003), trasciende la personalidad positiva y la emoción, puesto que añade una teología afirmativa o catafática, la cual reconoce y sostiene que es posible llegar a conocer a Dios a partir del contacto con la realidad y la razón, así mismo, Frankl (1994) expone que es vital las creencias religiosas o la fe de lo divino en la vida de los individuos, lo cual posibilite la trascendencia personal. Tanto Seligman como Frankl entienden que la meta o último peldaño de la felicidad, sentido de vida y finalidad de la existencia es Dios (Valhondo y Cadavieco, 2011).

\section{Sentido de vida como parte sustancial en la eficacia del proceso terapéutico en salud mental}

El sufrimiento deja de serlo en cierto modo cuando se halla un sentido, contrariamente, sufrir sin sentido produce desesperación (Frankl, 2004). Ante alguna afección mental, encontrar un sentido de vida podrá ayudar a afrontar de manera sana la situación, y bajo este enfoque es que se trabaja en la logoterapia, es decir, ayudando a hallar un sentido de vida que posibilite resistir sanamente el sufrimiento o las crisis, afrontando el miedo a la muerte, sin negarla, sino más bien, encarándola como un proceso normal y universal en todos los seres humanos. Desarrollar y darle la debida importancia a un proyecto de vida, en el cual estén 
inmersos nuestro contexto familiar, académico y profesional, posibilita que los individuos cuando están experimentando alguna crisis o inestabilidad emocional, puedan encarar y superar de una manera más positiva y valiente la situación en la que se encuentran inmersos, por lo tanto, la motivación intrínseca es un medio que enaltece la salud mental, y con mayor solvencia, en un proceso psicoterapéutico. Barripedro, Eraña y Mayol (2001) sostienen que, a partir de la despreocupación, la satisfacción personal, la sociabilidad, y mantenerse ocupados, favorece el fortalecimiento de salud mental.

Reflexionar y enriquecer nuestro sentido de vida, autoestima y esperanza previene que se genere un estado depresivo en la persona (Hedayati y Khazaei, 2014), por lo que estos tres factores refuerzan nuestro sistema inmunológico y evitan también diversas enfermedades psicosomáticas, lo cual será también un aliciente en un proceso psicoterapéutico, puesto que no solo se trabajará a nivel mental de una manera efectiva, sino que también, se verá contribuido por el bienestar a nivel físico y orgánico. La reflexión es una cualidad que poderosamente contribuye a la aquiescencia que una persona posee en lo tocante a cuán responsable es de su salud mental, al permitir erradicar pensamientos o posturas dañinas para consigo mismo como para con los demás, ya que enfatizará, producto de esa reflexión, en todo ese cúmulo de ideas que lo perturban y que, con ayuda de una profesional de la salud mental, como parte de un proceso psicoterapéutico, podrá llegar a desarrollar esa paz mental necesaria en nuestras vidas, según Ahumada (2013), el sentido de vida es fundamental para que el individuo se pueda autorrealizar, debido a que ello permitirá la autoeficacia y reflexión positiva de la existencia, estos sentimientos positivos evitarán la depresión y ansiedad.

Un aspecto significativo en cualquier individuo en el cual se aprecia la asociación del sentido de vida con la salud mental, es la resiliencia, puesto que esta capacidad enfrentar, superar y salir fortalecido de crisis, adversidades y dificultades de manera valiente, sana y positiva, resulta clave y es lo que permitirá involucrarse idóneamente en el proceso de sanación a nivel mental, de tal manera que, el poseer un sentido de vida representado por tener una meta, proyecto inspirador o un propósito superior alimenta esa resiliencia, la nutre y enriquece, puesto que es un aliciente que emerge a nivel mental en los momentos más difíciles de nuestras vidas, más aun en un tratamiento psicoterapéutico; conforme a lo que sostiene Escudero (2007), en cuanto al estudio de los pilares de la resiliencia, uno de los componentes internos importantes es la cualidad personal denominada sentido de vida, representada por la motivación intrínseca para lograr metas y objetivos, capacidad de automotivación, y superación de sentimientos desesperanzadores que pudiese surgir.

Vale decir que, el sentido de vida y el humor genera más resiliencia, lo cual posibilita afianzar el sentido de vida y contribuye notoriamente en la salud mental, aquellas personas que tienen menor resiliencia están mucho más expuestos a 
la ansiedad y el insomnio (González, 2014). Por lo tanto, uno de los pilares de la resiliencia es el humor, entendiendo un humor sano y enriquecedor que contribuya en gestar resiliencia que es un factor crucial hoy en día como parte del trabajo personal y social en cuanto a la prevención y promoción de salud mental, diversos entornos y organizaciones relacionadas a la salud vienen empoderando cada vez más esta capacidad. Finalmente, toda la suma de razones, ilusiones y acciones que motivan involucrarse con otras personas en un estado armonioso de sociedad, es lo que Velasco (2004), plantea como sentido de vida, y ese estado armonioso, contribuye cabalmente con la salud mental a nivel de una persona que está inmersa en cualquier proceso terapéutico, lo cual se extrapola a la salud mental a nivel social, como parte de las familias, sociedad y comunidad.

\section{CONCLUSIÓN}

Por todo lo expuesto, se concluye que poseer y enriquecer el sentido de vida en una persona posibilitará gestar y desarrollar salud mental, lo cual pueda ser transmitido o inspirado a nivel de la familia, sociedad o comunidad, velando principalmente en personas que se encuentran en un proceso terapéutico, confiriéndoles esa fortaleza propia de tener un propósito superior en la vida, el deseo de auto superación enfocado en factores intrínsecos, como la fe, los sentimientos, la conexión con la naturaleza y la vida. El vacío existencial presente en muchos individuos en diversas sociedades puede y debe ser erradicado enarbolando el sentido de vida, lo cual siente las bases de fortalecimiento a nivel de salud mental, ya que nadie está exento de vivir una crisis a nivel personal, emocional, familiar o hasta social a lo largo de su vida, pero, habrá una mayor eficacia para poder entenderlas y superarlas positiva y valientemente, más aún, por medio de adherirse correctamente a la terapia psicoterapéutica, si es que se ha alimentado y fortalecido a nivel de nuestras familias y sociedad el desarrollo y enriquecimiento de nuestro sentido de vida. Por último, entender que todos los seres humanos, a pesar de sus creencias $\mathrm{y}$ vicisitudes, poseen en realidad un profundo sentido religioso inconscientemente, el cual alimenta y fortalece nuestro sentido de vida, es un factor sustantivo y crucial cuando se aborda el tema de salud mental, puesto que esta búsqueda constante del significado último de nuestra vida es aquel diamante intangible, aquel lazo tácito que nos permite conectarnos con nosotros mismos, y por momentos efímeros y cruciales darnos cuenta de cómo es todo, cómo es la vida entera, y esos momentos son los más hermosos e inspiradores.

\section{REFERENCIAS}

Ahumada, G. (2013). Autoeficacia y felicidad en ingresantes a una universidad privada de Trujillo. Revista de Psicología,15(1), 24-36. 
Baleriano, J., Pinzón, D., Gaona-Pérez, E. y Hernández, D. (2015). Evaluación del juicio moral en estudiantes de psicología de la universidad INCCA de Colombia. Revista Evaluación e Intervención Psicológica REIP, 1(1), 26-33.

Barripedro, M., Eraña, I. y Mayol, L. (2001). Relación de la actividad física con la depresión y satisfacción con la vida en la tercera edad. Revista de Psicología del Deporte, 10(2), 239-246.

Bauman, Z. (2007). Vida de consumo. Argentina: Fondo de Cultura Económica.

Bauman, Z. (2008). El Arte de la Vida. Buenos Aires: Paidós.

Buber, M. (1969). Yo y tú. Buenos Aires, Argentina: Nueva Visión.

Escudero, J. (2007). Relación entre resiliencia y sentido de vida (religiosidad intrínseca y objetivos vitales) en adolescentes de cuarto y quinto de Secundaria. Tesis para optar el título profesional de psicólogo. Lima: Universidad Nacional Mayor de San Marcos.

Frankl, V. (1994). Logoterapia y análisis existencial. Textos de cinco décadas. Barcelona: Herder.

Frankl, V. (1999). El hombre en busca de sentido último. Barcelona: Paidós.

Frankl, V. (2004). El hombre en busca de sentido. España: Editorial Herder.

Frankl, V. (2011). La presencia ignorada de Dios. Psicoterapia y religión. Barcelona: Herder.

García, C. (2010). La vida es una moneda. El arte de vivir. Barcelona: Plataforma Editorial.

Gómez, R. (2013). Relación psicoterapéutica. Enfoque fenomenológico existencial. Lima: Fondo Editorial de la Universidad Inca Garcilaso de la Vega.

González, J. (2014). Salud mental y resiliencia en estudiantes de arte, Universidad de los Andes, Venezuela. Tesis doctoral de psiquiatría. Universidad Autónoma de Madrid: Madrid.

Gottfried, A. (2016). Adaptación argentina del PIL Test de Crumbaugh y Maholick [en línea], Revista de Psicología, 12(23).

Hedayati, M. y Khazaei, M. (2014). An investigation of the relationship between depression, meaning in life and adult hope. Procedia-Social and Behavioral Sciences, 114, 598-601.

Lukas, E. (2005). Una Vida Fascinante. Buenos Aires: Editorial San Pablo.

Luna, J. (2011). Logoterapia: un enfoque humanista existencial. Bogotá: Editorial San Pablo.

Martinez, E. (2012). El diálogo socrático en la psicoterapia. Colombia: SAPS (Sociedad para el Avance de la Psicoterapia Centrada en el Sentido).

Maslow, A. (2005). Motivación y personalidad. Madrid: Editorial Díaz De Santos.

Salomon, A. (2015). Encontrando y realizando sentido. Diálogo socrático y ejercicios vivenciales en logoterapia. Lima: Universidad Femenina del Sagrado Corazón. Ensayos e Psicología. 
Savater, F. (2007). Las preguntas de la vida. Barcelona: Ariel.

Seligman, M. (2003). La auténtica felicidad. Barcelona: Ediciones B.

Valhondo, A. y Cadavieco, J. (2011). El significado del paso de los años: claves y retos para un envejecimiento saludable y feliz. Internacional Journal of developmental and educational psychology,1(4), 633-643.

Velasco, S. (2004). El sentido de vida de los adolescentes: un modelo centrado en la persona. Tesis para obtener el grado de Maestra en Desarrollo Humano. México, Universidad iberoamericana.

Yalom, I. (1984), Psicoterapia Existencial. Barcelona: Herder. 